\title{
Noncommutative Quantum Hall Effect and Aharonov-Bohm Effect
}

\author{
B. Harms ${ }^{1, \text { * }}$ and O. Micu ${ }^{1, \oplus}$ \\ ${ }^{1}$ Department of Physics and Astronomy, The University of Alabama, Box 870324, Tuscaloosa, AL 35487-0324, USA
}

\begin{abstract}
We study a system of electrons moving on a noncommutative plane in the presence of an external magnetic field which is perpendicular to this plane. For generality we assume that the coordinates and the momenta are both noncommutative. We make a transformation from the noncommutative coordinates to a set of commuting coordinates and then we write the Hamiltonian for this system. The energy spectrum and the expectation value of the current can then be calculated and the Hall conductivity can be extracted. We use the same method to calculate the phase shift for the Aharonov-Bohm effect. Precession measurements could allow strong upper limits to be imposed on the noncommutativity coordinate and momentum parameters $\Theta$ and $\Xi$.

PACS numbers: 11.10.Nx, 73.43.-f
\end{abstract}

\section{INTRODUCTION}

Noncommutative theories arise in string theory [1, 2] and in the present search for quantum gravity [3], while Yang-Mills theories on non-commutative spaces [4] appear in string theory and M-theory. The noncommutative theories which are studied the most are the ones in which it is assumed that coordinates do not commute with each other. For more generality we will assume that the momenta are noncommutative as well. In the end if one wants to restrict these results to the case where only the coordinates are noncommutative, one can set the parameter that describes the noncommutativity of the momenta to zero.

We shall follow an approach [5] in which we will express the noncommutative coordinates $x_{i}, p_{i}$ as linear combinations of canonical variables of quantum mechanics $\alpha_{i}, \beta_{i}$. We will see that the noncommutativity will introduce additional terms in the Hamiltonian of the equivalent commutative description.

In the present work we are calculating modifications to the quantum Hall effect and to the Aharonov-Bohm effect in this noncommutative scenario. In the former effect an electric current flows through a conductor in a magnetic field which has a component perpendicular to the plane of the electron's trajectory. The magnetic field exerts a transverse force on the electrons which tends to push them to one side of the conductor. This is most evident in a flat and thin conductor where the magnetic field is perpendicular to the plane of the conductor. Charge accumulates at the sides of the conductors producing a measurable voltage between the two sides of the conductor. The case of charged particles in magnetic fields (the Landau problem) was previously considered in the literature from prospectives which differ from the one taken in the present work [6], [7], [8], [9], [10].

The Aharonov-Bohm effect emphasizes the fact that it is not the electric and magnetic fields but the electromagnetic potentials which are the fundamental quantities in quantum mechanics. In this effect a beam of electrons is split in two and the two beams follow two different paths. An interference pattern is produced when the two different beams of electrons recombine because there will be a phase shift between the two beams, and this phase shift depends on the magnetic flux enclosed by the two alternative paths. This phase shift is observed even if they pass through regions of space in which the magnetic field is null but the vector potential is not zero. The noncommutative Aharonov-Bohm effect was studied using the star product approach in [11], [12].

\footnotetext{
*Electronic address: bharms@bama.ua.edu

†Electronic address: micu001@bama.ua.edu
} 
In Section II we consider electrons which are moving on a noncommutative plane in the presence of an electric field in this plane and an external magnetic field which is perpendicular to the noncommutative plane. In the commutative case the experiment described above leads to the Hall effect. Once again, for more generality we assume both the coordinates (which now become operators) and momenta do not commute. We calculate corrections due to noncommutativity to the Hall conductivity, and we will show that in the limit when the parameters describing noncommutativity go to zero, we recover the commutative case. In Section III we calculate deviations due to noncommutativity to the phase shift for the Aharonov-Bohm effect. Also here we will show that in the commutative limit we reproduce the usual results. Section IV contains the limits on the noncommutativity parameters which we obtain from our analysis. In Section $\mathrm{V}$ we discuss the results of our analysis.

\section{NONCOMMUTATIVE QUANTUM HALL EFFECT}

\section{A. Hall effect}

An electron moving on the $(x, y)$ plane in a uniform electric field $\vec{E}=-\vec{\nabla} \phi$ and a uniform magnetic field $B$ which is perpendicular to the plane is described by the Hamiltonian

$$
H=\frac{1}{2 m}\left(\vec{p}+\frac{e}{c} \vec{A}\right)^{2}-e \phi,
$$

We will adopt the symmetric gauge (this gauge is well suited for the experiment described in the previous section)

$$
\vec{A}=\left(-\frac{B}{2} y, \frac{B}{2} x\right)
$$

and we will consider the scalar potential to be

$$
\phi=-E x
$$

If we substitute (2) and (3) into (11), we can write the Hamiltonian in the following form

$$
H(\vec{p}, \vec{r})=\frac{1}{2 m}\left[\left(p_{x}-\frac{e B}{2 c} y\right)^{2}+\left(p_{y}+\frac{e B}{2 c} x\right)^{2}\right]+e E x
$$

We want to calculate modifications to the Hall conductivity due to the effects of space noncommutativity. For generality, we assume a set of coordinates and momenta which satisfy the following commutation relations

$$
\begin{aligned}
{\left[x_{i}, x_{j}\right] } & =i \Theta_{i j}, \\
{\left[p_{i}, p_{j}\right] } & =i \hbar^{2} \Xi_{i j}, \\
{\left[x_{i}, p_{j}\right] } & =i \hbar \delta_{i j} .
\end{aligned}
$$

where $\Theta_{i j}$ and $\Xi_{i j}$ are antisymmetric matrices characterizing the noncommutativity of the phase space geometry.

Following the same treatment as [5] we define linear transformations from the set of noncommutative coordinates to a commutative set of canonically conjugate coordinates $\left(\alpha_{i}, \beta_{i}\right)$ which satisfy

$$
\begin{aligned}
{\left[\alpha_{i}, \alpha_{j}\right] } & =0, \\
{\left[\beta_{i}, \beta_{j}\right] } & =0, \\
{\left[\alpha_{i}, \beta_{j}\right] } & =i \hbar \delta_{i j} .
\end{aligned}
$$


The relation between the two sets of coordinates is defined as follows

$$
\begin{aligned}
& x_{i}=a_{i j} \alpha_{j}+b_{i j} \beta_{j} \\
& p_{i}=c_{i j} \beta_{j}+d_{i j} \alpha_{j}
\end{aligned}
$$

where a, b, c and d are in this case $2 \times 2$ transformation matrices. The relations (5), (6), (77) and (8)), (9), (10) determine the conditions which the transformation matrices must satisfy. In matrix form they are

$$
\begin{aligned}
\mathbf{a b}-b^{\mathbf{T}}-\mathbf{b a}^{\mathbf{T}} & =\frac{\Theta}{\hbar} \\
\mathbf{c d}^{\mathrm{T}}-\mathbf{d c}^{\mathrm{T}} & =-\hbar \Xi \\
\mathbf{c a}^{\mathbf{T}}-\mathbf{b d}^{\mathbf{T}} & =\mathbf{I}
\end{aligned}
$$

where $\boldsymbol{\Theta}$ and $\boldsymbol{\Xi}$ are antisymmetric matrices.

The transformation matrices are not unique (more details can be found in [5]) but a convenient choice for our purposes during the calculations that follow is to keep matrices a and $\mathbf{c}$ diagonal and single valued. In order to maintain the same number of free parameters, matrices $\mathbf{b}$ and $\mathbf{d}$ are chosen to be antisymmetric

$$
\begin{aligned}
a_{i j} & \equiv a \delta_{i j}, & c_{i j} & \equiv c \delta_{i j} \\
b_{i j} & \equiv b \epsilon_{i j}, & d_{i j} & \equiv d \epsilon_{i j}
\end{aligned}
$$

Equations (13,15) become

$$
\begin{aligned}
& a b=-\frac{\Theta}{2 \hbar} \\
& c d=\frac{\hbar \Xi}{2} \\
& a c-b d=1
\end{aligned}
$$

We solve for three parameters and we get

$$
\begin{aligned}
b & =-\frac{\Theta}{2 a \hbar} \\
c & =\frac{1}{2 a}(1 \pm \sqrt{\kappa}), \quad \kappa \equiv 1-\Theta \Xi \\
d & =\frac{\hbar a}{\Theta}(1 \mp \sqrt{\kappa})
\end{aligned}
$$

Substituting (11), (121) and (21), (22), (23) into our Hamiltonian (44), we can rewrite it in the following way

$$
H(\vec{\alpha}, \vec{\beta})=\frac{1}{2 m}\left[h_{1}^{2}\left(\alpha_{i}\right)^{2}+h_{2}^{2}\left(\beta_{i}\right)^{2}-h_{3} \epsilon_{i j} \alpha_{i} \beta_{j}\right]+a e E \alpha_{1}-\frac{\Theta}{2 a} e E \beta_{2}
$$

with

$$
\begin{aligned}
& h_{1}^{2}=a^{2}\left[\frac{\hbar}{\Theta}(1 \mp \sqrt{\kappa})-\left(\frac{e B}{2 c}\right)\right]^{2} \\
& h_{2}^{2}=\frac{\Theta^{2}}{4 \hbar^{2} a^{2}}\left[\frac{\hbar}{\Theta}(1 \pm \sqrt{\kappa})-\left(\frac{e B}{2 c}\right)\right]^{2} \\
& h_{3}=\left[\left(\frac{e B}{2 c}\right)^{2} \Theta+\hbar^{2} \Xi-\frac{\hbar e B}{c}\right]
\end{aligned}
$$


We make the following coordinate transformations

$$
\begin{aligned}
& \beta_{1} \rightarrow \beta_{1} \\
& \beta_{2} \rightarrow \beta_{2}-\frac{\frac{\Theta}{2 a} e E}{2 h_{2}^{2}}
\end{aligned}
$$

and the Hamiltonian will take the following form

$$
H(\vec{\alpha}, \vec{\beta})=\frac{1}{2 m}\left[h_{1}^{2}\left(\alpha_{i}\right)^{2}+h_{2}^{2}\left(\beta_{i}\right)^{2}+h_{3} \epsilon_{i j} \alpha_{i} \beta_{j}\right]+h_{4} \alpha_{1}-h_{5}
$$

where we have defined

$$
\begin{aligned}
& h_{4}= \pm 2 e E a \frac{\sqrt{\kappa}}{\Theta\left[\frac{\hbar}{\Theta}(1 \pm \sqrt{\kappa})-\left(\frac{e B}{2 c}\right)\right]} \\
& h_{5}=\frac{1}{2 m} \frac{e^{2} E^{2}}{4\left[\frac{\hbar}{\Theta}(1 \pm \sqrt{\kappa})-\left(\frac{e B}{2 c}\right)\right]^{2}}
\end{aligned}
$$

Now we have to discuss the eigenvalue problem

$$
\hat{H} \Psi=\mathcal{E} \Psi
$$

It is convenient to perform the change of variables [14]

$$
\begin{aligned}
\hat{z} & =\alpha_{1}+i \alpha_{2}, \\
\hat{p_{z}} & =\frac{1}{2}\left(\beta_{1}-i \beta_{2}\right) .
\end{aligned}
$$

We define two sets of creation and annihilation operators

$$
b^{\dagger}=-2 i h_{2} \hat{p}_{\bar{z}}+h_{1} \hat{z}+\lambda, \quad b=2 i h_{2} \hat{p}_{z}+h_{1} \hat{\bar{z}}+\lambda
$$

and

$$
d^{\dagger}=-2 i h_{2} \hat{p}_{\bar{z}}-h_{1} \hat{z}, \quad d=2 i h_{2} \hat{p}_{z}-h_{1} \hat{\bar{z}}
$$

where $\lambda=\mp m e E \sqrt{\kappa} / h_{3}$. These two sets of operators commute with each other and satisfy the following commutation relations

$$
\begin{aligned}
{\left[b, b^{\dagger}\right] } & =2 m \hbar \omega \\
{\left[d^{\dagger}, d\right] } & =2 m \hbar \omega
\end{aligned}
$$

with $\omega=h_{3} / m$.

In terms of these operators the Hamiltonian is

$$
\hat{H}=\frac{1}{4 m}\left(b b^{\dagger}+b^{\dagger} b\right)-\frac{\lambda}{2 m}\left(d^{\dagger}+d\right)-\frac{\lambda^{2}}{2 m}-h_{5}
$$

We observe that the Hamiltonian in (57) is composed of two mutually commuting parts

$$
\hat{H}=\hat{H}_{o s c}-\hat{H}_{1}
$$

We will calculate the eigenvalues $\mathcal{E}$ and the eigenfunctions $\Psi$ of the two commuting parts of the Hamiltonian separately. For the harmonic oscillator part

$$
\hat{H}_{o s c}=\frac{1}{4 m}\left(b b^{\dagger}+b^{\dagger} b\right)
$$


the eigenvalue value equation $\hat{H}_{o s c} \Phi_{n}=\mathcal{E}_{n}^{o s c} \Phi_{n}$ is easily solved and it leads to a discrete spectrum

$$
\begin{aligned}
\Phi_{n} & =\frac{1}{\sqrt{(2 m \hbar \omega)^{n} n !}}\left(b^{\dagger}\right)^{n} \mid 0>, \\
\mathcal{E}_{n}^{o s c} & =\frac{\hbar \omega}{2}(2 n+1), \quad n=0,1,2, \ldots
\end{aligned}
$$

The eigenvalue equation for $\hat{H}_{1} \phi_{\gamma}=\mathcal{E}_{\gamma} \phi_{\gamma}$ can be analyzed in terms of eigenvalues of the operators $\alpha_{i}$ and $\beta_{i}$

$$
\begin{aligned}
\phi_{\gamma} & =\exp \left(-i\left(\gamma \alpha_{2}+\frac{h_{1}}{\hbar h_{2}} \alpha_{1} \alpha_{2}\right)\right) \\
\mathcal{E}_{\gamma} & =\frac{\hbar \lambda h_{2}}{m} \gamma+\frac{\lambda^{2}}{2 m}+h_{5}, \quad \gamma \in \mathbb{R}
\end{aligned}
$$

which is a continuous spectrum.

We can now combine the two solutions and as a result, the eigenfunctions and energy spectrum of the Hamiltonian $\hat{H}$ are

$$
\begin{aligned}
\Psi_{(n, \gamma, \Theta, \Xi)}=|n, \gamma, \Theta, \Xi\rangle & =\frac{1}{\sqrt{(2 m \hbar \omega)^{n} n !}} \exp \left(-i\left(\gamma \alpha_{2}+\frac{h_{1}}{\hbar h_{2}} \alpha_{1} \alpha_{2}\right)\right)\left(b^{\dagger}\right)^{n} \mid 0> \\
\mathcal{E}_{(n, \gamma)} & =\frac{\hbar \omega}{2}(2 n+1)-\frac{\hbar \lambda h_{2}}{m} \gamma-\frac{\lambda^{2}}{2 m}-h_{5}
\end{aligned}
$$

\section{B. Hall conductivity}

The Hall conductivity can be calculated by means of the Hamiltonian $\hat{H}$ given above. We define the current operator $\hat{\vec{J}}$ on the noncommutative plane as

$$
\hat{\vec{J}}=\frac{i e \rho}{\hbar}[\hat{H}, \hat{\vec{r}}]
$$

The expectation values of the components of the current operator $\langle\hat{\vec{J}}\rangle$ calculated with respect to the eigenstates $|n, \gamma, \Theta, \Xi\rangle$ are

$$
\begin{array}{r}
\left\langle\hat{J}_{x}\right\rangle=0, \\
\left\langle\hat{J}_{y}\right\rangle=-e \rho \frac{1-\Theta \Xi}{\frac{B}{c}-\frac{e B^{2} \Theta}{4 \hbar c^{2}}-\frac{\hbar \Xi}{e}} E .
\end{array}
$$

Therefore the Hall conductivity on the noncommutative plane, which we denote by $\sigma_{H}$ is

$$
\sigma_{H}=-e \rho \frac{1-\Theta \Xi}{\frac{B}{c}-\frac{e B^{2} \Theta}{4 \hbar c^{2}}-\frac{\hbar \Xi}{e}}
$$

We notice that if the noncommutativity parameters $\Theta$ and $\Xi$ are taken to be equal to zero, we obtain the same value for the Hall conductivity as in the commutative case $\left(\sigma_{H}=-\rho e c / B\right)$

Using Eq.(52) we can look at particular cases derived from our result. In the noncommutative scenarios which are most commonly discussed in the literature, only the coordinates are noncommutative and the parameter $\Xi$ is equal to zero. In this case we obtain

$$
\sigma_{H}=-e \rho \frac{1}{\frac{B}{c}-\frac{e B^{2} \Theta}{4 \hbar c^{2}}}
$$


Alternatively we can imagine a noncommutative scenario in which coordinates commute but momenta are noncommutative. In this case also we obtain modifications of the commutative Hall effect due to the presence of the term proportional to $\Xi$

$$
\sigma_{H}=-e \rho \frac{1}{\frac{B}{c}-\frac{\hbar \Xi}{e}}
$$

From an experimental point of view, the last case that we consider might be much more important. Our result predicts that if space and momenta are noncommutative, even without the presence of an external magnetic field, the value of the Hall conductivity should be different from zero

$$
\sigma_{H}=e^{2} \rho \frac{1-\Theta \Xi}{\hbar \Xi}
$$

In this case the sign of the conductivity is different. If the sensitivity of the experiments is increased sufficiently, the effect might eventually be detected and it would be a clear signature of noncommutativity. There is one situation in which the Hall conductivity is still zero in this scenario, and that is if the two noncommutativity parameters $\Theta$ and $\Xi$ are naturally adjusted such that one is the inverse of the other. In this case the numerator of (55) would be equal to zero.

\section{NONCOMMUTATIVE AHARONOV-BOHM EFFECT}

We will use the same approach to study the Aharonov-Bohm effect in the noncommutative plane. We start from a similar Hamiltonian as in (1)

$$
H=\frac{1}{2 m}\left(\vec{p}+\frac{e}{c} \vec{A}\right)^{2}+V
$$

where $e$ is the charge on an electron.

Using the same gauge as in (2), the Hamiltonian becomes

$$
H(\vec{p}, \vec{r})=\frac{1}{2 m}\left[\left(p_{x}-\frac{e B}{2 c} y\right)^{2}+\left(p_{y}+\frac{e B}{2 c} x\right)^{2}\right]+V(\vec{x}),
$$

Following a similar derivation as in the previous part of the paper, we can rewrite $H$ once again in terms of $\alpha_{i}$ and $\beta_{i}$ as

$$
H(\vec{\alpha}, \vec{\beta})=\frac{1}{2 m}\left[h_{1}^{2}\left(\alpha_{i}\right)^{2}+h_{2}^{2}\left(\beta_{i}\right)^{2}-h_{3} \epsilon_{i j} \alpha_{i} \beta_{j}\right]+V(\vec{\alpha}, \vec{\beta})
$$

where the coefficients $h_{1}, h_{2}$ and $h_{3}$ are the ones defined in (25), (26) and (27). We use the fact that $2 h_{1} h_{2}=h_{3}$ and we rewrite the Hamiltonian once again as

$$
H(\vec{\alpha}, \vec{\beta})=\frac{1}{2 m}\left[\left(h_{2} \vec{\beta}-h_{1} \vec{\alpha}\right)^{2}\right]+V(\vec{\alpha}, \vec{\beta})
$$

where we have defined $\vec{\beta}=\left(\beta_{1}, \beta_{2}\right)$ and $\vec{\alpha}=\left(-\alpha_{2}, \alpha_{1}\right)$.

We now have to solve Schrodinger's equation

$$
\left[\frac{1}{2 m}\left(h_{2} \frac{\hbar}{i} \nabla-h_{1} \vec{\alpha}\right)^{2}+V(\vec{\alpha}, \vec{\beta})\right] \Psi=i \frac{\partial \Psi}{\partial t}
$$


where we substituted $\vec{\beta} \rightarrow \frac{\hbar}{i} \nabla$.

We write

$$
\Psi=e^{i g} \psi
$$

where

$$
g(\vec{\alpha})=\frac{h_{1}}{h_{2} \hbar} \oint \overrightarrow{\alpha^{\prime}} d \alpha^{\prime}
$$

Then

$$
\nabla \Psi=e^{i g}(i \nabla g) \psi+e^{i g} \nabla \psi
$$

but

$$
\nabla g=\frac{h_{1}}{h_{2} \hbar} \vec{\alpha}
$$

and using this we have from Eq.(63)

$$
\left(h_{2} \frac{\hbar}{i} \nabla-h_{1} \vec{\alpha}\right) \Psi=h_{2} \frac{\hbar}{i} e^{i g} \nabla \psi
$$

The first term in the (60) becomes

$$
\left(h_{2} \frac{\hbar}{i} \nabla-h_{1} \vec{\alpha}\right)^{2} \Psi=-h_{2}^{2} \hbar^{2} e^{i g} \nabla^{2} \psi
$$

If we substitute (61) and (66) into (60) we can see that $g(\vec{\alpha})$ is just a phase difference and it will be equal to

$$
\begin{aligned}
g(\vec{\alpha}) & =\frac{h_{1}}{h_{2} \hbar} \oint^{\alpha} \overrightarrow{\alpha^{\prime}} d \alpha^{\prime} \\
& =\frac{h_{1}}{h_{2} \hbar} \oint^{\alpha}\left(-\mathbf{i} \alpha_{2}^{\prime}+\mathbf{j} \alpha_{1}^{\prime}\right) d \alpha^{\prime} \\
& =\frac{h_{1}}{h_{2} \hbar} 2 \pi \alpha^{2}
\end{aligned}
$$

Substituting the expressions for the constants $h_{1}$ and $h_{2}$ from (25) and (26) we have for the phase shift

$$
g(\vec{\alpha})=\frac{2 a^{2}\left[\frac{\hbar}{\Theta}(1 \mp \sqrt{\kappa})-\left(\frac{e B}{2 c}\right)\right]}{\Theta\left[\frac{\hbar}{\Theta}(1 \pm \sqrt{\kappa})-\left(\frac{e B}{2 c}\right)\right]} 2 \pi \alpha^{2}
$$

We notice that if we take the noncommutativity parameters $\Theta$ and $\Xi$ to zero, only the solution with the upper signs is physical

$$
g(\vec{\alpha})=\frac{2 a^{2}\left[\frac{\hbar}{\Theta}(1-\sqrt{\kappa})-\left(\frac{e B}{2 c}\right)\right]}{\Theta\left[\frac{\hbar}{\Theta}(1+\sqrt{\kappa})-\left(\frac{e B}{2 c}\right)\right]} 2 \pi \alpha^{2}
$$

Moreover for $\Theta$ and $\Xi$ zero, setting the free parameter $a$ to 1 , gives the same phase shift as in the commutative case $-e \Phi / \hbar$ where by $\Phi$ is the magnetic flux. 
In the limit when $\Theta$ and $\Xi$ are small, we can expand $g(\vec{\alpha})$ to the first order in both parameters,

$$
g(\vec{\alpha})=\left(-\frac{e B}{c \hbar}+\Xi-\frac{e B^{2} \Theta}{4 c^{2} \hbar^{2}}\right) \pi \alpha^{2}
$$

Again if we consider the case for which only the coordinates are noncommutative but the momenta commute with each other $(\Xi=0)$, the phase shift becomes

$$
g(\vec{\alpha})=\left(-\frac{e B}{c \hbar}-\frac{e B^{2} \Theta}{4 c^{2} \hbar^{2}}\right) \pi \alpha^{2}
$$

Another scenario we can consider is the one for which the coordinates commute but the momenta are noncommutative. In this case we again obtain modifications of the commutative phase shift due to the presence of the term proportional to $\Xi$

$$
g(\vec{\alpha})=\left(-\frac{e B}{c \hbar}+\Xi\right) \pi \alpha^{2}
$$

For these two last cases that we studied, we observe that if the external magnetic field can be adjusted with enough sensitivity, we should find a value of the magnetic field for which the phase shift vanishes.

In the absence of an external magnetic field, again the phase shift is different from zero if the coordinates and the momenta are noncommutative. To see this we look back at equation (69) and we set the magnetic field to zero

$$
g(\vec{\alpha})=\frac{2 a^{2}(1-\sqrt{\kappa})}{\Theta(1+\sqrt{\kappa})} 2 \pi \alpha^{2}
$$

\section{EXPERIMENTAL LIMITS ON $\Theta$ AND $\Xi$}

The Hall conductivity (53) can be measured with an accuracy of one part in a billion. We can use this experimental limit to impose an upper limit of $10^{-34} \mathrm{~m}^{2}$ on the noncommutativity parameter $\Theta$. This limit on $\Theta$ is weaker by six orders of magnitude than the one imposed by [15] using data from experiments which test Lorentz invariance. Also the authors of [16] propose a stronger limit on theta by measuring differential cross sections for small angles in scattering experiments. However, the latter experiment is very difficult to perform because it requires the measurement of scattering angles between 1 and 2 degrees at energies of the order of $200 \mathrm{GeV}$. We can also impose a limit on $\Theta$ using Aharonov-Bohm measurements, but this limit is much weaker than the one that can be imposed using the Hall effect.

One of the advantages of our calculation is that we are able to consider the case when momenta are noncommutative, and we can also impose limits on the magnitude of the parameter which describes it. ¿From the experiments which measure the Hall conductivity we find that $\Xi$ must be smaller than $10^{-19} \mathrm{~m}^{-2}$. Also from (70) we can see that noncommutativity of momenta would induce a phase shift even in the absence of external magnetic fields. If the momenta are noncommutative and the phase shift could be measured with enough accuracy, a phase shift would be detected even in the absence of a magnetic field.

\section{DISCUSSION}

The electrons in the low-temperature Hall effect and in the Aharonov-Bohm effect do not interact appreciably with other particles, thus allowing a rather simple form for the Hamiltonian in each case. 
Although noncommutativity introduces some complexity into the expressions for the Hamiltonians in these two effects, they are still sufficiently simple that we were able to obtain wave vectors and energy eigenvalues or the phase shifts in closed form. This in turn allowed us to obtain expressions for the quantities measured in each of these two effects plus the deviations from the commutative forms of the quantities due to noncommutativity in analytical form. Several interesting features arise in the noncommutative forms of the quantum Hall effect and the Aharonov-Bohm effect. In the former effect the deviation of the conductivity due to noncommutativity is independent of the magnetic field to lowest order in the parameter $\Theta$ for $\Xi=0$. If the conductivity can be measured with sufficient precision, a deviation from the normal (magnetic field dependent) value would be circumstantial evidence for noncommutativity. In both the quantum Hall effect and the AharonovBohm effect deviations occur in the conductivity and phase shift respectively even if there is no magnetic field.

The limits which the two effects studied in this work can set on the $\Theta$ noncommutative parameter, while not as strong as the one set in [15], are nevertheless significant. The limit on the $\Xi$ parameter which was obtained in this work is the first one we have seen. The results reported on here suggest that high precision measurements in atomic and molecular systems may be able to rival or even exceed the strongest limit set by nuclear systems [15]. For this reason we are in the process of studying the Josephson effect using the method described above.

\section{ACKNOWLEDGEMENTS}

This research was supported in part by the University of Alabama's Research Advisory Committee.

[1] N. Seiberg and E. Witten, JHEP 9909, 032 (1999).

[2] N. Seiberg, L. Susskind, and N. Toumbas, JHEP 0006, 044 (2000).

[3] J. W. Moffat, Phys. Lett. B491,345 (2000).

[4] E. Witten, Surveys Diff. Geom. 7, 685 (1999).

[5] A. Smailagic and E. Spallucci, Phys. Rev. D 65, 107701 (2002).

[6] V. P. Nair and A. P. Polychronakos, Phys. Lett. B 505, 267 (2001).

[7] C. Duval and P. A. Horvathy, Theor. Math. Phys. 144, 899 (2005).

[8] A. Kokado, T. Okamura, T. Saito, Phys. Rev. D 69, 125007 (2004).

[9] B. Chakraborty, S. Gangopadhyay, A. Saha, Phys. Rev. D 70, 107707 (2004).

[10] P. A. Horvathy, M. S. Plyushchay, Nucl. Phys. B 714, 269 (2005).

[11] M. Chaichian, A. Demichev, P. Presnajder, M. M. Sheikh-Jabbari, A. Tureanu, Phys. Lett. B 527, 149 (2002); M. Chaichian, A. Demichev, P. Presnajder, M. M. Sheikh-Jabbari, A. Tureanu, Nucl. Phys. B 611, 383 (2001).

[12] B. Basu, S. Ghosh, S. Dhar, Europhys. Lett., 76, 395 (2006).

[13] A. Smailagic and E. Spallucci, J. Phys. A36, L517 (2003).

[14] O.F. Dayi, A. Jellal, J. Math. Phys.43, 4592 (2002).

[15] S. M. Carroll, J. A. Harvey, V. A. Kostelecky, C. D. Lane, T. Okamoto, Phys. Rev. Lett. 87,141601 (2001).

[16] H. Falomir, J. Gamboa, M. Loewe, F. Mendez, J.C. Rojas, Phys.Rev.D 66, 045018 (2002). 